\title{
Ulrich van Loyen, Napoli sepolta. Viaggio nei riti di fondazione di una città
}

Meltemi, Milano, 2020

Francesco Faeta

\section{OpenEdition}

\section{Journals}

Edizione digitale

URL: http://journals.openedition.org/aam/3637

DOI: $10.4000 /$ aam.3637

ISSN: 2038-3215

Editore

Dipartimento Culture e Società - Università di Palermo

Notizia bibliografica digitale

Francesco Faeta, «Ulrich van Loyen, Napoli sepolta. Viaggio nei riti di fondazione di una città», Archivio antropologico mediterraneo [Online], Anno XXIII, n. 22 (2) | 2020, online dal 31 décembre 2020 consultato il 27 janvier 2021. URL: http://journals.openedition.org/aam/3637 ; DOI: https://doi.org/ 10.4000/aam.3637

Questo documento è stato generato automaticamente il 27 janvier 2021.

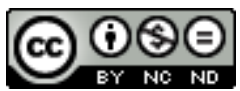

Archivio antropologico mediterraneo è distribuita con Licenza Creative Commons Attribuzione - Non commerciale - Non opere derivate 4.0 Internazionale. 


\section{Ulrich van Loyen, Napoli sepolta. Viaggio nei riti di fondazione di una città}

Meltemi, Milano, 2020

Francesco Faeta

NOTIZIA

ULRICH VAN LOYEN, Napoli sepolta. Viaggio nei riti di fondazione di una città, traduzione dal Tedesco di Massimo De Pascale, fotografie di Anja Dreschke, Meltemi, Milano, 2020. ISBN: 9788855192248 
1 David Foster Wallace, nel suo stimolante (e faticoso) romanzo Infinite Jest, utilizza un neologismo, che indica un concetto complesso, quello di experialismo. Proverò a soffermarmi su tale concetto in via preliminare; mi si perdoni quella che apparentemente potrà sembrare una digressione, ma credo che il quadro concettuale che vi si traccia sarà utile per collocare criticamente lo stimolante lavoro di Ulrich van Loyen. Un lavoro molto sfaccettato, che prende in analisi fenomeni anche assai diversi tra di loro, che necessita, a mio avviso, di un punto prospettico centrale per essere compreso appieno; punto prospettico che l'autore rinviene nell'unità territoriale, culturale e sociale cui diamo il nome di Napoli. Tutto ciò che viene messo in scena nelle quasi

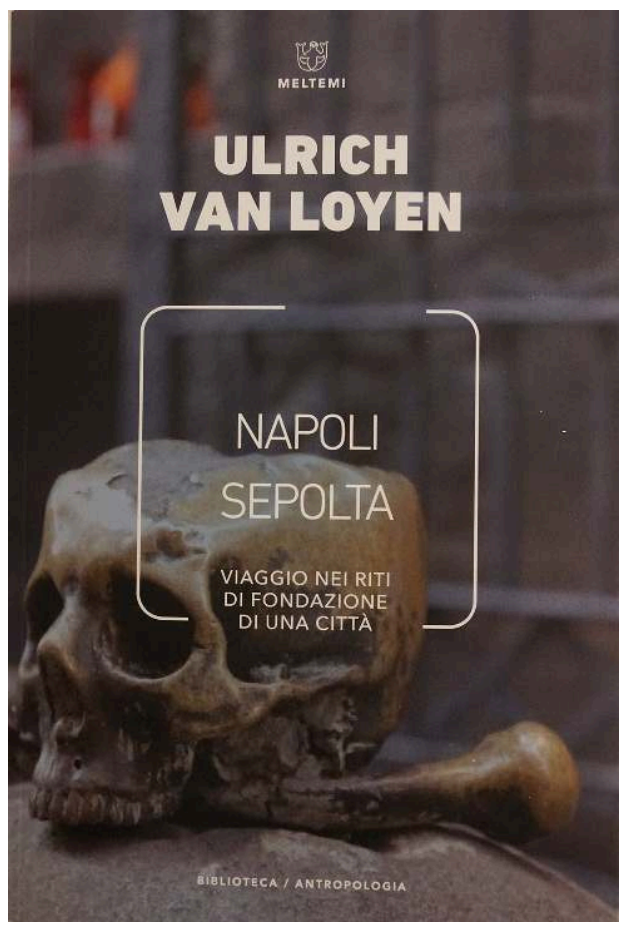
quattrocento pagine del suo volume esiste perché un'entità cogente gli conferisce senso e un imprescindibile significato unitario («non una città qualsiasi, ma questa città, in cui tutte le altre categorie [simboliche] perdono valore», p. 326; «una città che [...] ricorda instancabilmente di essere più di una semplice città», p. 339). Ma perché quest'unità cogente possa sostenere al meglio il suo ruolo ermeneutico ed epistemico, senza scivolamenti negli scenari rassicuranti del folklore, del senso comune e delle rappresentazioni di genere (cosa da cui l'autore si tiene lontano), occorre capire perché essa è cogente, per quale curvatura moderna e contemporanea della sua storia, in altre parole, essa è così; perché Napoli è Napoli insomma.

2 Per experialismo, come lo scrittore l'ha pensato e come io ulteriormente lo declino a fini interpretativi, s'intende qualcosa di opposto (e complementare, in realtà) all'imperialismo. Se quest'ultimo indicava la volontà di annessione territoriale dello Stato, al fine del dominio politico, dello sfruttamento economico, dell'ottimizzazione del rapporto di dipendenza e dell'esercizio di un potere inderogabile, il primo indica invece la tendenza a cedere sovranità rispetto alle aree territoriali considerate più problematiche, delegando ad altri Stati o a enti sussidiari la loro gestione, espellendole di fatto dal confine nazionale e imperiale e lasciandole in una loro indefinita e fluida marginalità e autarchia. Questa condizione garantisce allo Stato experialista di non avere a che fare con i grattacapi, le contraddizioni sociali, i costi e gli oneri di mediazione politica, i provvedimenti relativi all'ambiente e alla sicurezza dei territori scomodi, utilizzandoli come discariche, sia in senso ambientale, sia per quel che concerne la più generale geopolitica, sia infine in termini di democrazia. Se quest'ultima costituisce un problema in un territorio dato, tale territorio può essere buttato fuori dal recinto nazionale e imperiale, in modo che la contaminazione rappresentata dalla sua crisi democratica non si estenda. Può essere, anzi, devoluto proprio a quelle forze e a quelle agenzie che provocano la crisi del regime democratico (se la sbrighino loro). Naturalmente, ciò non vuol dire che ogni potere presente 
nell'area experiale sia sempre e del tutto consenziente rispetto al processo (né che tutti i cittadini lo condividano senza critiche e resistenze). E che quindi tali poteri non tentino, a loro volta, un movimento di infiltrazione nei gangli del sistema centrale, anche per implementare il proprio margine di delega e di profitto all'interno della realtà in cui vivono. $\mathrm{Ma}$, più frequentemente, il fenomeno, concedendo al territorio experializzato ampi margini di autonomia, pur nella dipendenza, crea consenso; e crea élite experiali astute e allineate, che traggono tornaconto dalla situazione.

3 Bene, l'experialismo a me sembra la modalità di gestione, da parte dello Stato nazionale, di vaste aree del Mezzogiorno italiano tra le quali si può annoverare l'area metropolitana di Napoli (così come, del resto, l'assai prossima terra dei fuochi). È discarica ambientale, innanzitutto, al cui interno le più rigide regole che valgono per il suolo patrio non hanno corso; è regione dove si forma e prospera un'élite impresentabile, che sostituisce quella che dovrebbe garantire la continuità nazionale; è luogo di fatto amministrato da un potere non legittimo, né ufficialmente riconosciuto, ma tollerato nel fare, nell'imporre ordine e gerarchia, nel garantire i margini del profitto coloniale; è spazio dove la democrazia che vige all'interno dello Stato, è del tutto disattesa, diviene mera rappresentazione di facciata, sostituita dalla legge della violenza, dell'inganno, dell'estorsione, dell'intimidazione, della corruzione e della clientela. La criminalità organizzata governa il territorio experializzato, in modo tale $\mathrm{da}$ consentire il margine di profitto tacitamente concesso al momento dell'estromissione. $\mathrm{E}$ lo Stato deposita in esso le sue scorie infette, consente che esso sia saccheggiato in tutte le sue stremate risorse, non pretende di esercitare se non un finto controllo sull'ambiente, sui beni culturali, sulla scuola, sulla sanità, sull'occupazione. Inserisce i suoi garanti amministrativi nel territorio secondo le regole elettive e di consenso che caratterizzano un potere delegato. Lascia che siano messi a tacere, nel territorio, i dissidenti, con i modi e nelle forme che il potere effettivo deciderà, $\mathrm{e}$ talvolta, quando è strettamente necessario, interverrà direttamente a garantire che la protesta resti sommessa. Si priva, lo Stato experialista, dell'onere di organizzare modernamente i trasporti e le infrastrutture, di far funzionare le scuole e gli ospedali, di imporre un dialogo costruttivo con i sindacati, di garantire la corretta erogazione dei servizi di welfare, di asfaltare le strade, di assicurare l'ordine (se non nei termini di mero contenimento), di illuminare le città, e così via.

È in un contesto politico e sociale di tal fatta, a mio avviso, che si pone la vasta indagine di van Loyen. Perché dentro situazioni desertificate secondo le logiche che ho riassunto sopra, nelle quali «tutti fanno affari con la camorra, tutti sono parte della camorra» (p. 50), si devono collocare nuovi istituti, sistemi di relazione, modalità di normalizzazione dei rapporti, figure di mediazione, ideologie della ricostruzione. In più passi del suo libro, lo studioso ricorda l'assenza dello Stato, o la sua perversa debolezza, nello scenario di Napoli: «quando sono arrivato io - scrive - [2013, nda] l'idea che lo Stato potesse costituire la risorsa indispensabile per la sopravvivenza della città era definitivamente tramontata» (p. 61), mentre il declassamento sociale complessivo e l'esclusione si fondavano imprescindibilmente sull'esperienza di uno Stato inaffidabile (p. 309). L'armamentario offerto dalla religiosità popolare, dunque, va a riempire questa zona desertificata, con un insieme di simboli, cerimonie, riti, esperienze esistenziali, sogni, significati reconditi o coperti, poetiche e politiche di strada che largamente contribuiscono a riempirla e a fornire un senso alla vita delle masse di diseredati e sottoproletari, molti dei quali inseriti più o meno organicamente nei circuiti criminali. Al centro di quest'universo significante vi è il culto delle anime 
purganti quale si esprime nei cimiteri ipogei e negli spazi religiosi cittadini, in particolare del cosiddetto rione Sanità. Van Loyen, chiarendo bene come tale culto abbia una funzione utilitaristica, fondando una prassi di relazione biunivoca tra vivi e defunti, lo descrive efficacemente, con dovizia di particolari, intrecciando il piano della restituzione critica con quello dell'autocitazione dai suoi taccuini di terreno, con un'ottima resa narrativa (anche se qualche passaggio appare non perfettamente risolto, ritengo per via della traduzione). Ma soprattutto il culto dedicato alle capuzzelle, ai teschi anonimi cui viene restituita identità e devozione attraverso un lungo e complesso lavoro di costruzione culturale e sociale - assai noto in passato e ben studiato; su uno di questi studi dovrò tornare più avanti -, una prassi liminare per eccellenza in una città contraddistinta per l'autore da una permanente, ciclica, morfologicamente varia e ricorrente condizione liminare, viene posto in una relazione organica con altri elementi. Van Layen ha il merito di evidenziare una rete di fenomeni (quelli che, nell'interpretazione che propongo per leggere in chiave di economia politica il suo lavoro, vanno a riempire il vuoto causato dall'experialismo), contraddistinti dal tratto della liminarità, ponendo in relazione (sullo sfondo) l'universo dei femminielli e (in primo piano) quello dei Battenti o Fujenti della Madonna dell'Arco. Cosa unifica tali fenomeni? La loro liminarità, come ho scritto, ma più concretamente il fatto che tale liminarità consente transiti e mediazioni molteplici: tra i generi (maschile-femminile), tra i vivi e i morti, tra gli universi malavitosi e le attività della vita di ogni giorno.

In particolare nell'analisi, a partire da una sorta di iniziale aria di famiglia per scendere poi in un'approfondita analisi di carattere strutturale, delle relazioni di somiglianza intercorrenti tra culto delle anime purganti e pellegrinaggio-processione verso il santuario della Madonna dell'Arco a Sant'Anastasia, si chiariscono forme e modelli della costruzione sociale intermedia (il mondo di mezzo), che ampiamente sostiene la vita sociale delle classi popolari nella Napoli contemporanea, secondo van Loyen. Sia il culto delle anime purganti sia la costruzione delle compagnie di penitenti e delle loro performance rituali, poggiano sulla sfera funebre. Da un lato i teschi; dall'altro le immagini «votive» di defunti, spesse volte in modo tragico o violento, poste a ragione sociale dei sodalizi e raffigurate sulle loro bandiere rituali. Queste figure pescate dalla sfera funebre contribuiscono a ricreare le famiglie (e qui s'inseriscono utilmente le osservazioni accessorie sui femminielli), su una base di nuova aggregazione sociale e culturale. La famiglia naturale e nucleare, spazzata via dal vuoto pneumatico lasciato dall'assenza dello Stato e delle consuete agenzie di mediazione, abbandonata in una condizione di permanente emergenza (la città come crisi permanente è immagine che l'autore adopera), deve ricrearsi come famiglia sociale, allargata, scelta, costruita. I teschi divengono allora, attraverso il culto che si sviluppa al loro intorno, il fulcro di più vaste aggregazioni familiari, di relazioni, di scambio e di prestazioni ineguali. Il riconoscimento di una sorta di antenato comune fonda prospettive di reciprocità e alleanza. Un processo analogo a quello che accade per la costituzione di una compagnia dedita al culto della Madonna dell'Arco, un culto che si esprime, prima, su base locale, di vicinato, rione, quartiere, e poi su una base territoriale più vasta, pur se sempre circoscritta, nella giornata cruciale del pellegrinaggio verso il santuario. Anche qui, attraverso la comune assunzione di un morto, si allarga l'iniziale, ristretta, certe volte precaria per via dell'indigenza degli adepti, per via della frammentazione sociale e religiosa dei contesti, base sociale, formando catene di alleanza che concorrono alla formazione della compagnia (una formazione che richiede una certa solidità sociale e 
una certa solvibilità economica). Ovviamente, e con più puntuale riferimento alla realtà del quartiere da lui studiato (nel quale l'autore sembra aver trovato un suo modus vivendi, non esente da piacere e divertimento, spendendo bene l'immagine stereotipica del Tedesco buono e un po' ingenuo), le relazioni che così si costruiscono, mentre consentono la vita individuale e di gruppo, costituiscono il principale veicolo di espressione e di affermazione delle reti clientelari e di radicamento del potere camorristico sul territorio. Per dirla con Foster Wallace, e con la mia lettura applicata delle sue nozioni, costituiscono il modo concreto in cui si riorganizza la vita sociale nei territori marginalizzati dalla cessione dello Stato. $\mathrm{E}$ in questa prospettiva la ricerca di van Loyen si sofferma su una miriade di episodi concreti attraverso i quali viene tessuta la trama delle nuove relazioni di sopravvivenza. Il culto dei morti sconosciuti a Napoli, in definitiva, lungi dall'essere un pittoresca sopravvivenza di un passato più o meno remoto, s'inserisce nel vivo della costruzione della città moderna o post-moderna.

6 Tale culto, tuttavia, e del resto le pratiche rituali legate all'esistenza del santuario di Sant'Anastasia, hanno una vita piuttosto lunga, si radicano in episodi storici concreti e in forme devozionali di lungo periodo, come il libro non manca di ricordare. Ciò sembrerebbe smentire la loro modernità o post-modernità e l'interpretazione di essi quali strumenti per la coesione sociale delle masse subalterne nel presente che van Loyen assegna loro. In effetti non è così. Le ossa dei morti oggi venerate, e la loro stessa antica venerazione, provengono da giacimenti che sono risultato di altre immani catastrofi (pestilenze, colera, vaiolo), di altre circostanze in cui si è creato, nella storia della città, un vuoto, una rarefazione della vita sociale, una disgregazione, bisognosa di reintegrazione secondo modelli metafisici e metastorici. E il culto dedicato alla Madonna dell'Arco, a sua volta, si basa su episodi di morte, di scacco, di desertificazione, di punizione, su una temporanea sospensione delle regole della pietas e su una contemporanea affermazione di un potere divino vendicativo e violento (non a caso van Loyen pone in evidenza il tratto brutale del culto odierno, che convive accanto a quello acrobatico e di destrezza, così come l'ostentazione delle pistole giocattolo di plastica sulle bancarelle, poste accanto alle immaginette della Madonna, lungo tutto il percorso del pellegrinaggio). Anche ieri, come innumerevoli episodi riportati da cronisti e studiosi testimoniano, il culto era legato alla strada, vista come metafora di un vuoto, come spazio anomico contrapposto alla casa. Oggi lo studioso rileva come esso sia essenzialmente appannaggio dei guaglion' 'e miezz' 'a strada e osserva, in un passaggio intenso della sua monografia, che «un fascino particolare della strada per $\mathrm{S}$. Anastasia sta nel potere straniante di questo paesaggio, che appartiene ancora alla città ma in cui diventa comprensibile la vita selvaggia che ha impregnato numerose biografie dei fedeli. È una classica zona di marioli: qui arrivano da Bari i convogli carichi di sigarette di contrabbando e di notte le prostitute africane e i femminielli si scaldano attorno al fuoco. I rifugiati subsahariani, dimenticati dai media e dalle organizzazioni [...] si spostano in silenzio verso le loro baracche. Come la maggior parte delle strade abbandonate, questa è deserta solo in apparenza: nella topografia di chi vi transita, quasi ogni metro è segnato con precisione e occupa una posizione nella rete delle attività quotidiane» (p. 308).

7 Ho prima scritto che sarei tornato su uno degli studi dedicati al culto delle anime purganti. È quello pubblicato nel 1993, ma basato su esperienze di terreno di anni precedenti, di Stefano De Matteis e Marino Niola. Il lavoro di van Loyen mi ha obbligato a rileggerlo e gliene sono grato perché ho avuto modo di apprezzarlo ancor più che nelle occasioni passate. Ciò che questa lettura comparata rivela è il diverso orizzonte 
culturale e scientifico con cui si guarda oggi alla realtà devozionale e rituale del Mezzogiorno italiano e la diversa compattezza delle narrazioni. De Matteis e Niola forniscono un ritratto sapiente, di estrema raffinatezza, che disegna le ragioni culturali, colte e popolari, del fenomeno osservato, ponendo in secondo piano, o per meglio dire lasciando all'approccio storico, la decifrazione dell'ambiente sociale in cui il culto si colloca (e ciò malgrado i cospicui documenti di natura antropologica che vengono addotti). La camorra non c'è nel loro racconto, e non certamente perché non fosse all'epoca presente sul territorio, ma perché il tipo di sguardo che l'antropologo produceva sulla realtà cerimoniale e rituale del Sud non la contemplava (come non contemplava la mafia o la 'ndrangheta). La storia culturale dell'area indagata è concepita, da un lato, come «segmentaria», con la continua fluttuazione tattica e strategica dei rapporti tra classi egemoni e classi subalterne, dall'altra come un continuum organico (nella sua diversità). La narrazione è compatta e assertiva, basata su unità semantiche incalzanti, guidate da una tesi di fondo esplicita e forte. Van Loyen, invece, punta soprattutto a comprendere il nesso che lega determinate formazioni culturali ai processi di strutturazione della società. Nel suo saggio, la camorra ha la stessa importanza delle anime purganti. È essa stessa testo e pre-testo (precondizione del testo). La sua narrazione, inoltre, rinuncia alla compattezza, ambisce a essere giustapposta, fluida, dialogica, problematica. Significativamente, nel testo di De Matteis e Niola le fotografie, raccolte al centro, opere di forte impatto autoriale di Antonio Biasucci (che continuerà a lavorare ancora sui cimiteri ipogei e sul loro culto), non rappresentano persone, se non incidentalmente, e neppure pratiche; quelle di Anja Dreschke, presenti nel libro di van Loyen, di basso profilo autoriale, impaginate in testo, sono cariche di un intento documentale, colme di pratiche, gesti, comportamenti, personaggi, relitti, spezzoni di strutture urbanistiche e architettoniche. Tra i due testi, infine, vi è anche una notevole diversità per quel che concerne la letteratura su cui poggiano: a differenza del primo studio, che si regge essenzialmente su una produzione italiana, francese, anglo-americana (con le ovvie eccezioni di autori quali a esempio Walter Benjamin o Otto Rank divenuti classici ineludibili della riflessione internazionale), tipica del paesaggio intellettuale italiano negli anni in questione (e ancor oggi), il Bildungsroman di van Loyen si basa anche su un apparato ermeneutico da noi meno noto e condiviso (Max Weber, naturalmente; Thomas Hautschild, per le indicazioni relative alla postura di terreno, per la consapevolezza della modernità del magismo, per il concetto di riserva culturale; Thomas Belmonte, per l'analisi della circolarità culturale tra classi superiori e inferiori; Alfred Sohn-Rethe, e il suo Ideal des Kaputten come strumento di lettura del comportamento sociale a Napoli; Theodor Trede per le sue analisi sull'evangelismo in città, cui l'autore dedica attenzione in molti suoi passaggi; Erhard Schuttpelz, per le sue letture demartiniane e per la sua prospettiva relativa ai processi di visualità; Italo Pardo e Jason Pine, per le loro perspicue prospettive di studio di aspetti importanti del making do in Naples, tra numerosi altri, a conferma della relativa peculiarità delle basi interpretative dell'antropologia tedesca contemporanea). Un richiamo assai frequente, infine, il libro opportunamente fa al lavoro intellettuale dei due mentori di terreno, in buona misura, dell'autore, il regista Luigi Di Gianni, e il già ricordato De Matteis. 